\title{
Congenital insensitivity to pain syndrome accompanied by neglected orthopedic traumas and complications
}

\author{
Recep Kurnaz ${ }^{*}, 1$, Murat Asci $^{2}$, Orhan Balta ${ }^{2}$, Kursad Aytekin $^{3}$, Taner Gunes ${ }^{1}$ \\ ${ }^{1}$ Department of Orthopedics and Traumatology, Acibadem Eskisehir Hospital, Eskisehir, Turkey, \\ ${ }^{2}$ Department of Orthopedics and Traumatology, School of Medicine, Gaziosmanpasa University, \\ Tokat, Turkey, ${ }^{3}$ Department of Orthopedics and Traumatology, School of Medicine, Giresun \\ University, Giresun, Turkey
}

\begin{abstract}
Pain is a protective mechanism. Congenital insensitivity to pain syndrome is a very rare disease in which there is no ability to feel physical pain. It has been reported that it occurs with an incidence of 1 in 125 million newborn. Patients with congenital insensitivity to pain may have various orthopedic complications such as recurrent fractures, osteomyelitis and neuropathic joints. The most frequently affected body parts are lower extremities. Besides, curvature of spine can be seen. Injuries in epiphyseal points may cause incompatibility of extremity. Charcot joints may develop, which can lead to neuropathic arthropathy as a result of insensitivity to pain. Here, we present a patient with traumatic fracture-dislocation on left hip that neglected the treatment, had a bilateral femur fracture and then had septic arthritis of knee.
\end{abstract}

Keywords: CIPA, Congenital insensitivity to pain syndrome, orthopedic complications

\section{Introduction}

Congenital insensitivity to pain syndrome (CIPA) is a very rare case in which there is no ability to feel physical pain. It has been reported that it occurs with an incidence of 1 in 125 million newborn. Therefore, very little is described in the literature about this devastating condition that both the patient and the family can suffer [1]. These patients do not feel pain in any trauma or injuries throughout their life. They have feeling of hot and cold. Pain is a protective mechanism. Because of lack of pain, bruises, bone fractures, and other health problems may go undetected in such people.

Received: January 2017; Accepted after review: March 2017; Published: March 2017.

*Corresponding author: Recep Kurnaz, MD, Department of Orthopedics and Traumatology, Acibadem Eskisehir Hospital, Eskisehir, Turkey.

E-mail: recepkurnaz@gmail.com
Small children with congenital insensitivity to pain may damage themselves by biting their corner of mouth or fingers. These repeated injuries often lead to a lower life expectancy. It is considered as peripheral neuropathy because it affects the peripheral nervous system.

Here, we present a patient with traumatic fracture-dislocation on left hip, who neglected the treatment, had a bilateral femur fracture and then had septic arthritis of knee.

\section{Case report}

An 8 year-old girl applied with pain and swelling in her left knee. Two years ago, the patient underwent osteosynthesis surgery with a diagnosis of right femur shaft fracture after trauma with reduction titanium elastic nail at another center. The implants were removed one year after the operation. She applied to the orthopedic physician with a complaint of 
pain in the left hip after a fall again 1.5 years ago. The patient who had a traumatic hip dislocation after the acetabulum fracture, was diagnosed with congenital hip dislocation and was not intervened (Figure 1). However, the hip was in place, when we looked at the older $\mathrm{x}$-rays of the patient. The patient, who had a left femur shaft fracture 1 year ago, underwent closed reduction and fixation with titanium elastic nail at another center (Figure 2).

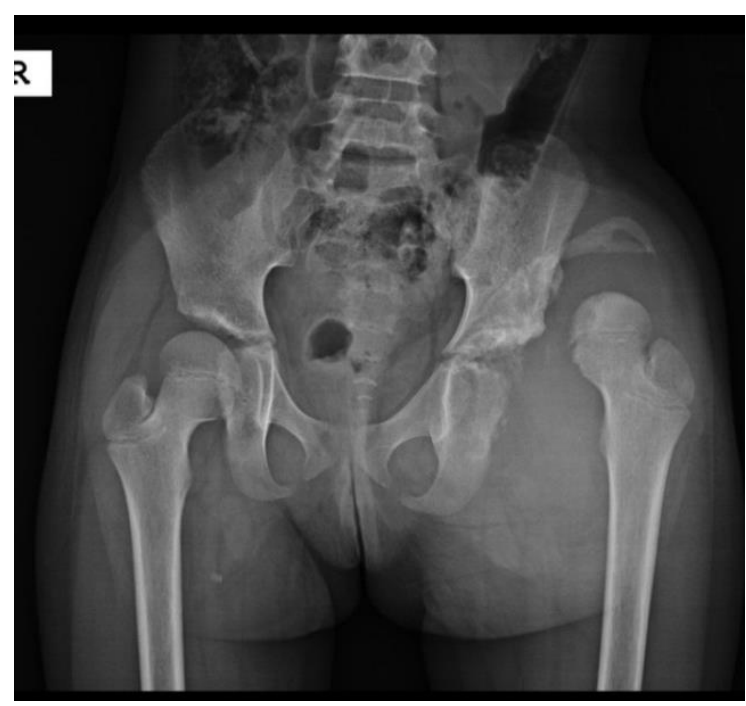

Fig. 1. Untreated traumatic hip dislocation which was thought as congenital hip dislocation
Destruction of the proximal tibial epiphyses and premature partial occlusion occurred due to the infection after the operation (Figure 3-4). Swelling in the left knee and increase in temperature was observed in the examination of the patient. After the investigations performed, septic arthritis was seen in the left knee. Two titanium elastic nails on the left femur were removed and joint debridement was performed (Figure 4).

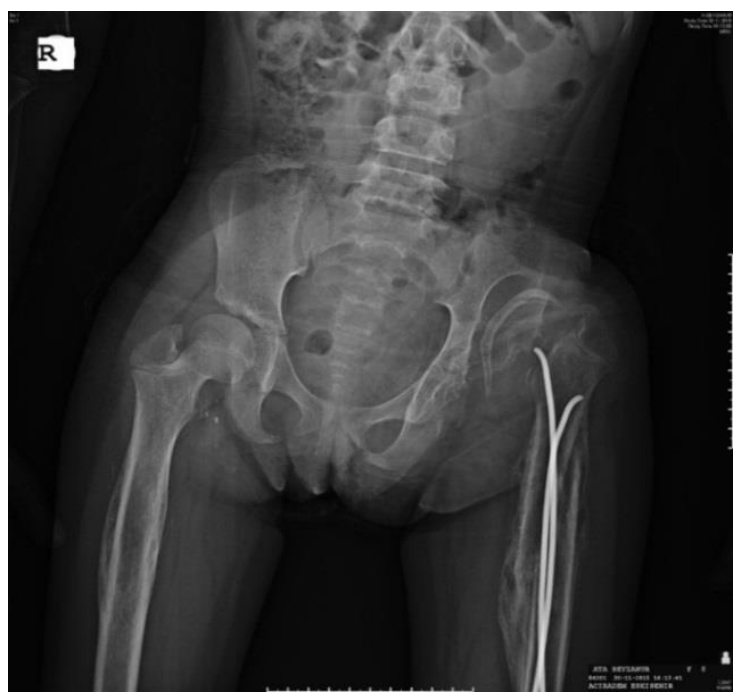

Fig. 2. Operated left femur fracture
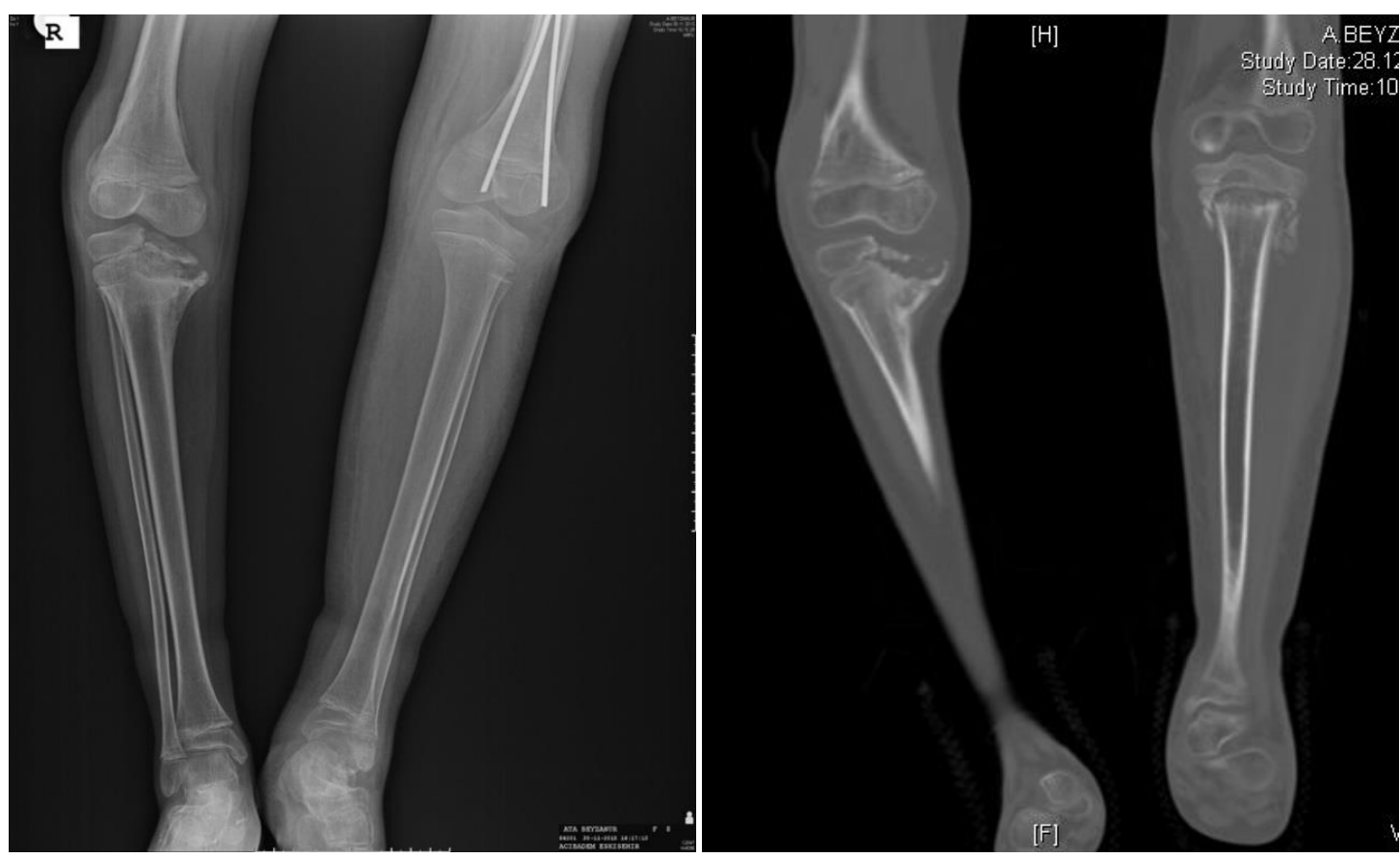

Fig. 3 and 4. Destruction of the proximal tibial epiphyses and premature partial occlusion 
There was no medical history in the patient's family. Her parents were healthy, and no consanguinity was present. Her APGAR score was 6 at one minute and 9 at five minutes. When she was born, there was no hypotonia, hypoxia, hypothermia or hyperthermia. When hospital records were examined, erythrocyte sedimentation rate (ESR) and C-reactive protein (CRP) values

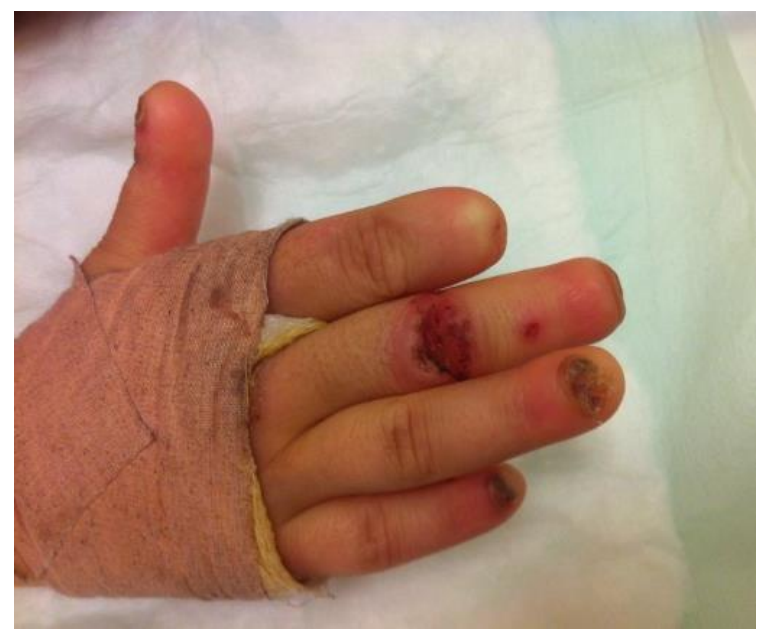

Fig. 5. Wounds due to fingers biting were observed to be consistently high. The family said she had had febrile seizures during infancy and they had been warned by the nurses because of not crying of the patient after the injection for the treatment. When looking at the patient's fingers, it was seen that she injured herself by biting her fingertips and some of 2, 3 and 4. Distal phalanges were missing (Figures 5, 6, and 7).

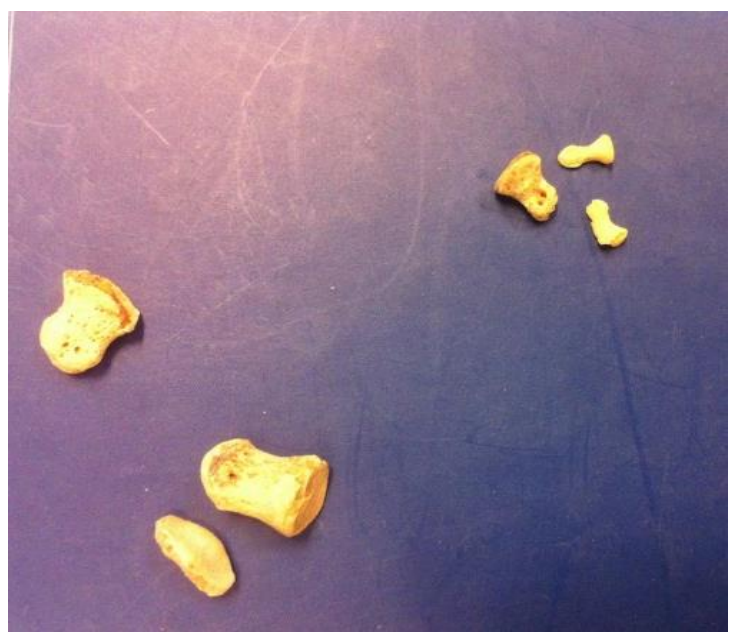

Fig. 6. Finger bones

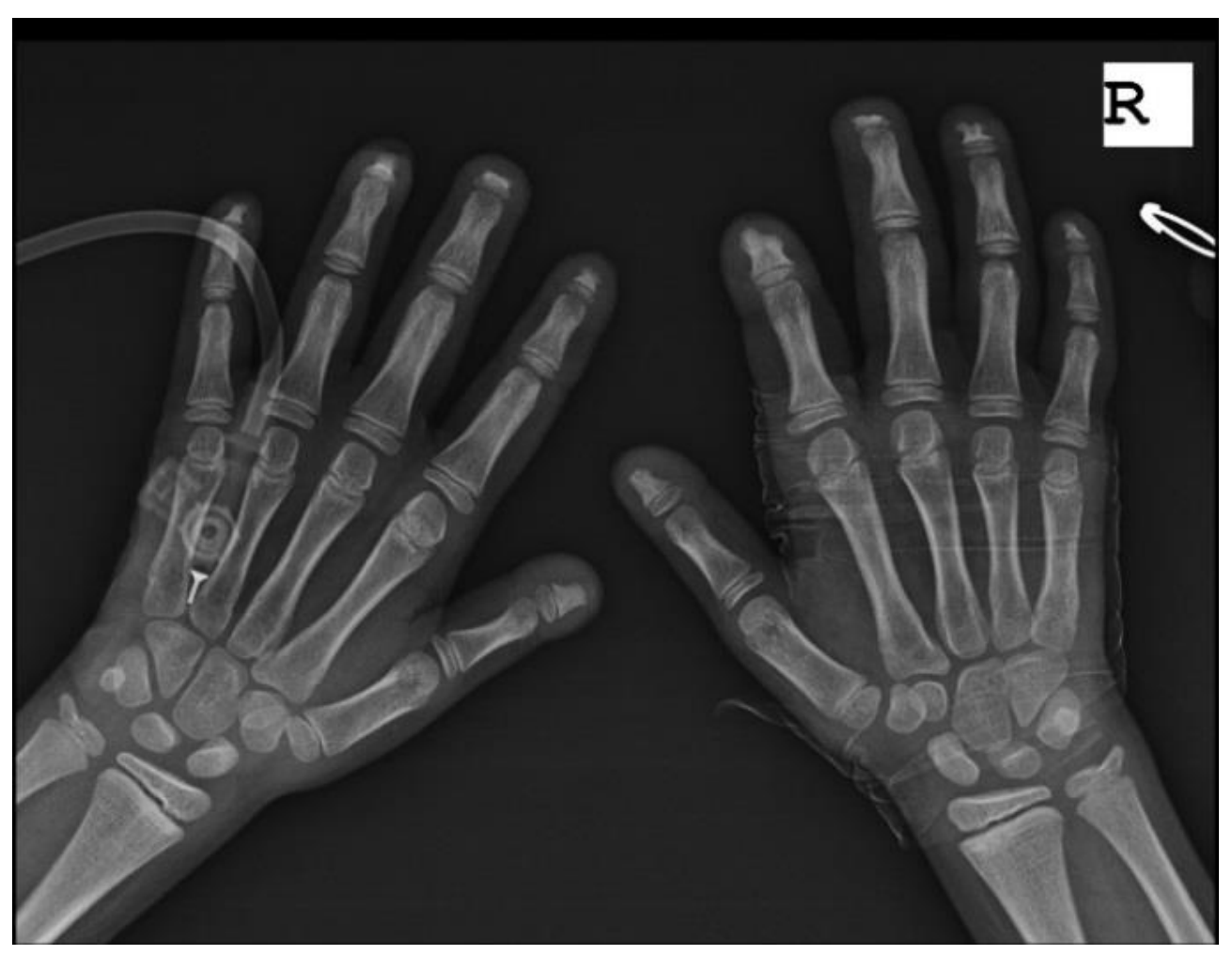

Fig. 7. The hands X-ray showing lack of distal phalanges due to fingers biting 
It was understood that she has a feeling of hot and cold but it is not very effective. However, it was noticed that she perceives the temperature while drinking tea. We learned from the family that her skin is dry, she never

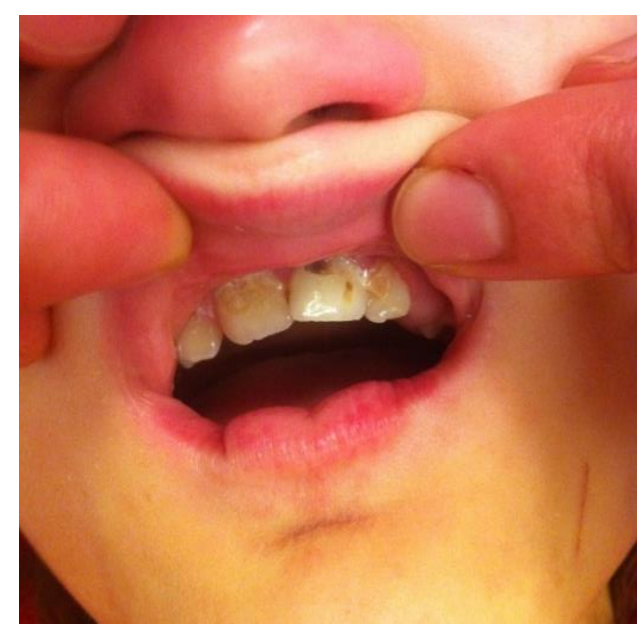

Fig. 8. Wounds in the corner of the mouth due to lips biting

\section{Discussions}

Insensitivity to pain may have different underlying causes such as syringomyelia, diabetes mellitus, leprosy and interstitial hypertrophic neuritis [1]. Insensitivity to pain can lead to various complications such as infection, ulceration and sometimes death [2].

Inheritance is divided into five categories based on clinical features, disease progression, autonomic involvement and specific genetic abnormalities in hereditary sensory autonomic neuropathy [3, 4]. Clinical evaluation of each subtype is necessary in the treatment and prevention of long-term complications. Type 1 (sensory radicular neuropathy) is characterized by sensory disorder particularly in lower extremity in the second and fourth decades of life. Patients often apply with foot joint complications, such as plantar ulcers, soft tissue infections and osteomyelitis. Type 2 (Morvan's disease) affects all four extremities and occurs during infancy or early childhood, a strong precursor for motor fibers. Patients usually have pathological fractures and sacral anhidrosis. Deep tendon reflexes are not present. Type 3 (Riley eDay Syndrome) occurs in the postnatal sweats and she took her teeth out herself (Figures 8 and 9). The difference of the case we presented is that there is no mental retardation.

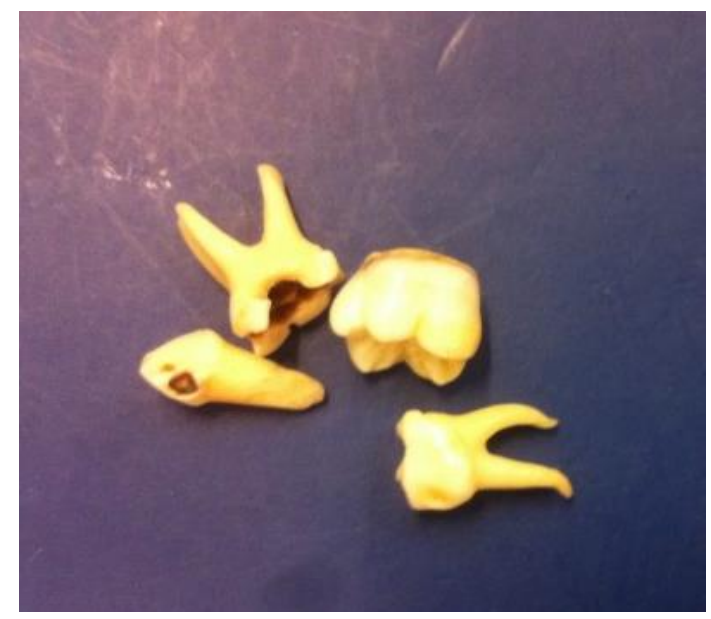

Fig. 9. The self-extracted teeth

period with nutritional failure, recurrent aspiration pneumonia and severe autonomic dysfunction, often ending up with dead in premature birth. Type 5 is characterized by the beginning of insensitivity to pain and temperature, and variable autonomic involvement in childhood [3].

CIPA was first described by Dearborn in 1932 and systematically published by Swenson in 1963 [5]. This extremely rare autosomal recessively inherited disease is described as hereditary sensory autonomic neuropathy type 4. Nerve fiber endings or electrophysiological studies should be assessed microscopically in order to distinguish congenital insensitivity to pain from peripheral neuropathic pains such as diabetes [6].

Diagnosis is confirmed with the aid of genetic tests whereas it can be diagnosed clinically. Thrush et al. suggested using three basic clinical features for diagnosis: the feeling of pain does not exist after birth, the entire body is affected, and all other sensory types and deep tendon reflexes can be used [7].

In addition to clinical evaluation for CIPA diagnosis, pharmacological tests (1:10.000 histamine intradermal reaction) and 
neuropathological examination in electron microscopy can also be used. In neurophysiologic examination, isolated axonal sensory polyneuropathy was determined in CIPA patient [8]. A histamine test is performed by intradermal injection of a $1 / 10.000$ solution of $0.05 \mathrm{ml}$ histamine phosphate. Generally, a hole surrounded by an erythematous flare occurs extending from 1 to $3 \mathrm{~cm}$ in a normal response; but in a pathological response, there is no flare and the pain is minimal.

Patients with congenital insensitivity to pain may have various orthopedic complications such as recurrent fractures, osteomyelitis and neuropathic joints. The most frequently affected body parts are lower extremities. Besides, curvature of spine can be seen. Injuries in epiphyseal points may cause incompatibility of extremity [9]. Charcot joints may develop, which can lead to neuropathic arthropathy as a result of insensitivity to pain [10].

Mostly weight-bearing joints, particularly knee and ankle joints, are affected. Despite fracture healing, arthropathy is progressive and eventually results in deformity and joint instability. In addition, cases such as autoamputation of fingers and toes have been reported in the literature. Unexplainable fever and anhydrous, another component of CIPA, can be fatal in some children [11].

The most common complaint in CIPA after birth is recurrent fever secondary to autonomic dysfunction and anhydrous [12]. This is the reason of $20 \%$ of deaths under 3 years of age. The most important characteristic is the selfmutilation behavior which causes oral ulceration in lip, tongue and cheeks, selfextraction of teeth, and biting one's own fingers.

Mutations in the NTRK1 (TRKA) gene affect CIPA. NTRK1 is located on chromosome 1 (1q21-q22) and encodes for autophosphorylated neurotrophic tyrosine kinase receptor type 1 in response to nerve growth factor [13]. However, these patients do not feel pain due to the mutation of the gene encoding the neurotrophic tyrosine kinase receptor.

In rodent studies, the presence of NGF was shown on osteosynthetic cells and the disruption of NGF led to the production of new hypertrophic bone $[14,15]$. This can cause significant bone hypertrophy in young patients. Patients with CIPA may develop recurrent, non-healing fractures with significant periosteal reactions and excessive callus formation, and $50 \%$ of patients have osteomyelitis and destruction of underlying bone [16].

We reviewed the literature searching for CIPA cases and possibly similar cases. Syringomyelia can lead to the resemblance of pain clutching, therefore leading to differential diagnoses [17]. Cornelia de Lange syndrome (CdLS) is a rare genetic syndrome with clinical findings related to multiple organ systems such as extremities, gastrointestinal tract, skin and central nervous system [18]. Lesch-Nyhan syndrome (LNS) is a rare $\mathrm{X}$-linked genetic disorder in which hypoxanthine-guanine phosphoribosyl transferase (HPRT) enzyme is absent clinically characterized by mental retardation, choreoathetosis, spasticity, hyperuricemia and cerebral palsy [19].

The other diagnoses were excluded after the genetic study which detected mutations in the NTRK1 gene responsible for CIPA in 2015.

The medical history, clinical signs of anhidrosis, pain insensitivity, genetic tests, and negative histamine flare tests of our patient sufficed to endorse the diagnosis of CIPA

Sixteen cases about CIPA have been reported in the literature. Makari et al. described two brothers with CIPA who suffered from child abuse [20]. In recent days, two cases of CIPA patients who continue treatment have been reported [21]. Generally, effective CIPA treatment has been established within the framework of family education and patient education [22]. Early detection of the disease using techniques such as prenatal genetic screening and amniocentesis is the only way to prevent the birth of an affected child, because treatment is not available [23]. After a child is born with CIPA, prevention of joint diseases is very important. Small cracks can be treated with conservative approaches. In a highly degenerated joint, arthrodesis may be appropriate eventually, but nonunion and infection are common. 


\section{Conclusion}

Currently, there is no standard treatment for CIPA patients. The most important factors are early diagnosis and being patient under the control of the family members to increase the quality of life by protecting from self-injury and other harms. Paying particular attention on patients, who has had extremity injury, can prevent complications and deformities; thus, the quality of life of the patients can be improved.

\section{References}

1. Ravichandra KS, Kandregula CR, Koya S, Lakhotia D. Congenital insensitivity to pain and anhydrosis: diagnostic and therapeutic dilemmas revisited. Int $J$ Clin Pediatr Dent 2015; 8:75-81.

2. van den Bosch GE, Baartmans MG, Vos $P$, Dokter J, White T, Tibboel D. Pain insensitivity syndrome misinterpreted as inflicted burns. Pediatrics 2014; 133:e1381-e1387.

3. Dyck PJ, Ohta M. Neural atrophy and degeneration predominantly affecting peripheral sensory neurons: In Peripheral Neuropathy, Dyck PJ, Thomas PK, Lambert $\mathrm{EH}$, editors. Toronto: WB Saunders Co 1975; 2:791-812.

4. Nagasako E, Oaklander A, Dworkin R. Congenital insensitivity to pain: an update. Pain 2003; 101:213e9.

5. Swanson AG. Congenital insensitivity to pain with anhydrosis. A unique syndrome in two male siblings. Arch Neurol 1963; 8:299-306.

6. Sandroni P, Martin DP, Bruce BK, Rome JD. Congenital idiopathic inability to perceive pain: anew syndrome of insensitivity to pain and itch with preserved small fibers. Pain 2006; 122:210-215.

7. Thrush DC. Congenital insensitivity to pain. A clinical, genetic and neurophysiological study of four children from the same family. Brain $J$ Neurol 1973; 96:369-386.

8. Kissani N, Krrati $\mathrm{H}$, Alarcon G, Belaaidi $\mathrm{H}$, Ouazzani R. Congenital insensitivity to pain: clinical and neurophysiological study in three sisters of a Moroccan family. Arch pediatr 2013; 20:1219-1224.

9. Bronfen C, Bensahel H, Teule JG. Orthopedic aspects of congenital insensitivity to pain. Chir Pediatr 1985; 26:193-196.

\section{Conflict of interest}

The author(s) declare that they have no competing interests.

\section{Consent}

Written informed consent was obtained from the patient's parents for publication of this case report and accompanying images.

10. Ali N, Sharma S, Sharma S, Kamal Y, Sharma S. Congenital insensitivity to pain with anhidrosis (HSAN type IV), extremely rare syndrome that can be easily missed by bone and joint surgeons: a case report. Iran J Pediatr 2012; 22:559-563.

11. Guidera KJ, Multhopp H, Ganey T, Ogden JA. Orthopaedic manifestations in congenitally insensate patients. J Pediatr Orthop 1990; 10:514-521.

12. Gupta B. Congenital insensitivity of pain with anhidrosis; clinical brief. Indian J Pediatr 2003; 70(1):109e11.

13. Melamed I, Levy J, Parvari R, Gelfand EW. A novel lymphocyte signaling defect: trk $A$ mutation in the syndrome of congenital insensitivity to pain and anhidrosis (CIPA). J Clin Immunol 2004; 24:441-448.

14. Asaumi $K$, Nakanishi $T$, Asahara $H$, Inoue $H$, Takigawa M. Expression of neurotropins and their receptors (TRK) during fracture healing. Bone 2000; 26:625e33.

15. Grills BL, Schuijers JA. Immunohistochemical localization of nerve growth factor in fractured and unfractured rat bone. Acta Orthop Scand 1998; 69:415e9.

16. Schulman $H$, Tsodikow V, Einhorn M, Levy $Y$, Shorer Z, Hertzanu Y. Congenital insensitivity to pain with anhidrosis (CIPA): the spectrum of radiological findings. Pediatr Radiol 2001; 31:701e5.

17. Magee KR, Schneider RC. Syringomyelia. Loss of deep-pain sensation with otherwise normal sensory perception. JAMA 1967; 200:795-796.

18. Grados MA, Alvi MH, Srivastava S. Behavioral and psychiatric manifestations in Cornelia de Lange syndrome. Curr Opin Psychiatry 2017; 30(2):92-96. 
19. Cauwels RG, Martens LC. Self-mutilation behaviour in Lesch-Nyhan syndrome. J Oral Pathol Med 2005; 34(9):573-575.

20. Makari GS, Carroll JE, Burton EM. Hereditary sensory neu- ropathy manifesting as possible child abuse. Pediatrics 1994; 93:842-844.

21. Karthikeyan M, Sreenivas T, Menon J, Patro DK. Congenital insensitivity to pain and anhydrosis: a report of two cases. J Orthop Surg 2014; 21:125-128.
22. Zhang $Y$, Haga N. Skeletal complications in congenital insensitivity to pain with anhidrosis: a case series of 14 patients and review of articles published in Japanese. J Orthop Sci Off $J$ Japanese Orthop Assoc 2014; 19:827-831.

23. Jerath NU, Shy ME. Hereditary motor and sensory neuropathies: understanding molecular pathogenesis could lead to future treatment strategies. Biochim Biophys Acta 2015; 1852:667-678. 